\title{
Research on the Tourism Toilet Layout for Sichuan Mountain Resort based on Graph Theory
}

\author{
Qiaoyi Li \\ Sichuan Vocational and Technical College, Suining Sichuan 629000, China.
}

\begin{abstract}
With rapid development of the mathematical modelling applications, the combination with other scenarios has been the trend. In the paper, we conduct research on the tourism toilet layout for Sichuan mountain resort based on graph theory. The toilet is necessary in the people life as it is to measure the social civilized degree of a window. Tourism scenic area in the toilet should first meet people's need to function, the small building, it is not the main landscape architecture, but necessary for visitors; Secondly, it should meet people's aesthetic needs, modelling beauty, design carefully and try to make it become a part of the scenery tourist area. Under this basic need, we conduct research on the issues theoretically and propose our novel perspective on the future trend.
\end{abstract}

Keywords: Layout; graph theory; tourism toilet; Sichuan; mountain resort; literature review.

\section{Introduction}

Tourism toilet refers to the tourist places, mainly for the tourists to the construction of service of the public toilet mainly distributed in the domestic tourist attractions, travel and other tourist reception site. Tourism toilet according to different tourist places, can be divided into is located in the city of the tourism, tourist center, scenic spots, tourist center, tourism shopping stores, places of entertainment, sports leisure places, tourist transportation, traffic on the way of break points, bus terminal, highway service area, tourist restaurants, tourist blocks the place such as a public toilet [1].

The toilet in the eyes of modern people has many symbolic meanings. For tourism, toilet represents the civilization degree of the tourism destination and the scientific management level potential market about the choice of the tourist destination. Tourism toilet location choice, first of all should follow the following basic principles. (1) Deployment in tourist areas or scenic tourist concentrated area. Tourist or visitors are concentrated in the scenic spot, generally are: main body construction, accent, square, museum, open-air movie theater, stadium, swimming pools, children's playground, etc. Here, visitors and more close, and stay a long time, without the toilet, will bring inconvenience, even affect visitors. (2) Deployed in the tourist area or around the scenic spot of import and export. Tourist area or the import and export of the scenic spot, stream, and flow to highly concentrated distribution of space and time in arrangement of tourism toilets, easy to visitors, control the population and the neighborhood area. At the same time, for the tourists visit scenic spot of the biological preparations. (3) Highlight the convenience. Tourism toilet decorate should not hinder the view, must be easy to find again at the same time, convenience and accessibility. Therefore, must be evenly distributed in the tourist area of each function which will determine the user experience.

Research are increasingly found in the real world of interactions between the actual network node importance degree and the effect is not the same, has the complexity and diversity, therefore, must be in the right to network research, on the basis of further research evolution of the weighted network, capturing and reveal the real network in order to further the connection that between the dynamic characteristics and the topology structure, and the change of weight of network evolution features or produced the important influence of system function. Therefore, further perfection and development of network theory of the hybrid model is one of the most important directions in the research of basic network model. Numerous studies have found that many of the actual network with the small-world characteristics and scale-free properties, but not so, but transition between the different characteristics of the complex. Under this background, in this paper, we conduct research on the tourism toilet layout for Sichuan mountain resort based on graph theory. In the figure 1, we show sample distribution of the tourism toilet, and in the later sections, we will discuss the issues in detail [2]. 


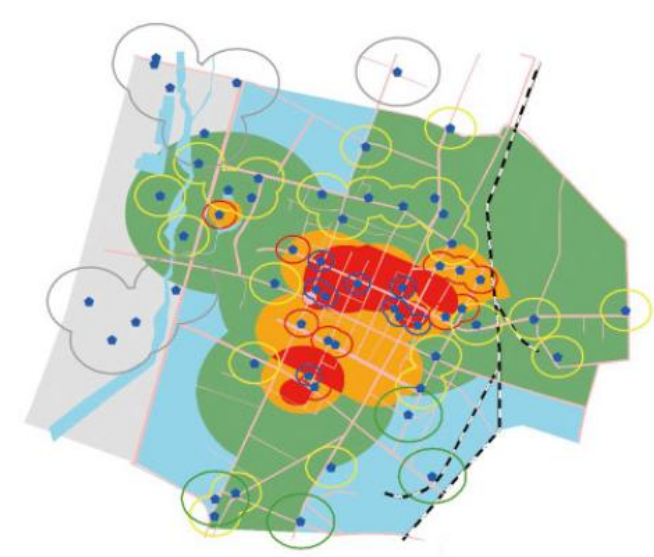

Figure 1. The Sample Distribution of the Tourism Toilet

\section{Our Designed Methodology}

\subsection{Sichuan Tourist Area and the Characteristics.}

Sichuan culture developed with celebrities, cultural relics all over Sichuan, extremely rich cultural tourism resources, and high efficience, grade, obvious advantages. Rich variety of cultural landscape and the natural landscape history for Sichuan culture tourism development laid a solid foundation. Sichuan is an important component part of the western development, and numerous advantages, especially the development of the tourism resources, unique taste in most western provinces, and has a leading and the important strategic position. Because of its long history and colorful minority culture, Sichuan is rich in cultural tourism resources is also varied.

Tourist area of infrastructure including roads, transportation, hotel, restaurant, parking, auxiliary facilities of scenic spot, communication, water and electricity, etc., in terms of the ecological tourist area, the above aspects are weak. Of all the infrastructure, roads and traffic are the breakthrough to should be a priority. Tourism is different from other industries, which is characterized by cash flow rolling and ecological tourist area in Sichuan province original natural scenery and the diverse ethnic culture and a cool summer climate, to attract urban population is self-evident [3].

Sichuan a profound history and culture goes back to ancient times, all over the world have a special liking to Bashu culture, we should not only well inherit the historical and cultural essence in Sichuan, and to innovation as continuous development generation of advanced culture, ancient civilization and modern civilization, mutual confluence, high level to show the brilliant Bashu culture, it is in Sichuan to further open to the world, let the world to learn more about an important part of Sichuan province.

\subsection{Features of the Mountain Tourist Area.}

Of the environmental quality and landscape resource security is the foundation of the tourism sustainable development. Due to the development, mountain tourism resources development, tourism activities, and the general mountain tourism destination itself characteristics, water loss and soil erosion that has been becoming the current mountain one universal environmental problems of tourism destination development. Tourism destination is advantageous to development of soil and water conservation ecological tourism industry to promote local economy.

A regional tourism development depends on the condition of the tourism resource value of high and low, the advantages and disadvantages and the combination of the regional economic background is good or bad. In a different way of combination, as develop differently. Although tourism resources for tourism development is crucial, but the regional tourism development is depend on basic resources, location and the combination of the regional economic background that rather than merely tourism resources. According to tourism area has a phenomenon of "compensation" optimal theory, tourism area in the compensation factor optimal compensation to the tourism resource development district.

\subsection{Graph Theory.}

Any one includes some kind of binary relation system can use the method of graph theory to analyze, and it has the characteristics of image directly. The application of graph theory in the 
graphics and geometric graphics is different as each edge can be assigned to right, form a weighted graph, used to studying the characteristics of the system, decision analysis, to determine the optimal design. Graph theory model/discrete model is established in the electromagnetic field of the basic laws of physics, thus directly from physical picture to establish algebraic equations, so the method profoundly embodies the "field" and "road" the combination of the two methods of analysis. Graph theory model is the application chart of the topological properties that combined with field structure characteristics and boundary conditions as shown in the figure 2 [4].

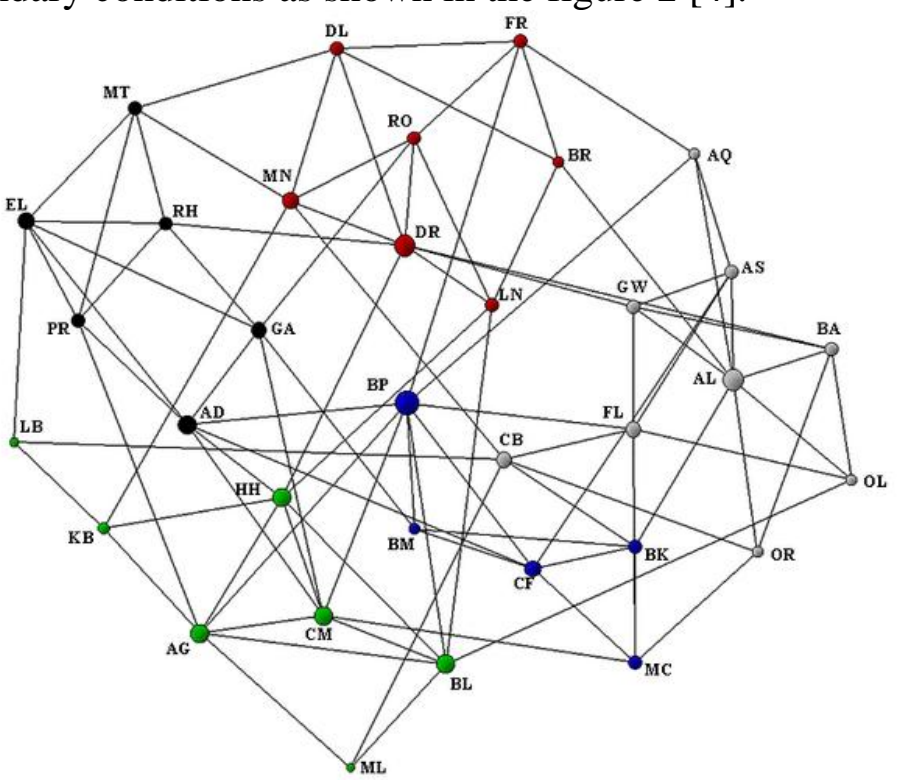

Figure 2. The Systematic Architecture of the Graph Theory

Different theoretical system of graph theory model is likely to have a completely different effect, as this also is underlined the importance of choosing appropriate theoretical system. Sometimes there may be multiple seem to choose the right model, carefully distinguish between them only subtle but critical difference that can choose the suitable for solving the model.

$$
G=\langle V, E\rangle \rightarrow(u, v)=(v, u)
$$

As illustrated in the formula one, we define the graph pattern. Graph theory algorithm plays an important role in computer science, it provides a lot of problems modeling of the system is simple and effective way. It can also be understood as a basic operation and regulation of operation the order form the complete problem-solving steps, or as designed in accordance with the requirements of the exact calculation sequence. In the formula two, we show the sample algorithm.

$$
l(s) \leftarrow(s, 0), l(v) \leftarrow(s, \infty)
$$

When using the graph theory model to solve the problem, we often encounter such a situation: after easily set up a model, but don't know how to analysis, to solve, or to build the model cannot show an important properties of the prototype. The weight for the nodes can be expressed as follows.

$$
\operatorname{Weight}(p, Z)=\sum_{n=1}^{N_{k}}\left[\exp \left(-d\left(p_{i}, p_{j}\right)\right)\right]
$$

In a study object, the object and there are many factors related to these elements are not necessarily consistent with our goal, in order to highlight the elements, which is closely linked with the goal to reduce the complexity of the thinking and we have to give up part of the elements. In the process of the modeling, ignore the prototype with solving the elements of target has little to do, to simplify the problem properly. But every coin has two sides, and if the simplified too degrees, it will make model becomes inaccurate, even is not correct, therefore, only with "brief" and find a balance between the "accurate", to set up the model which has the best effect.

\subsection{Suggestions for the Tourism Toilet Layout.}

In general, the processing technique of the tourism toilet layout there are three main types: basic concealment, fusion and beautification. The processing technique mainly from meet the needs of the tourist function, the combination of both tourism and the scenic toilet and coordination, and on the 
basis of the correlation of basic tourism toilet and scenery, consider visitors appreciate the taste and aesthetic idea, because of the different time and space, and adjust measures to local conditions to take tourism toilet layout method. Under the prior discussion on the graph theory, we propose the listed suggestions [5].

- Supporting type layout. In general scenic spots, often can only highlight a major landscape, the visitors to stay focused and observe a place for a long time. So in the arrangement of tourism toilet, it can go to the meet the need. Most scenic spots, the use of more edge to the layout of the hidden form, because in supporting type layout, tourism scenic area in general is not big, although of edge and hidden, as long as the sign design skillfully as visitors or easy to find.

- Scattered point layout. In larger traveling scenic area, or visitors more scenic spots, the general arrangement of three or more tourism toilet, as can satisfy the needs of tourists. A total of more than a tourism toilet decorate a form in the scenic area, often form a point. Based on triangle para relations, on the vertices of a triangle as the tourism toilet decorate place, to the whole triangle forming control pattern, often the center of gravity is located in scenic spots layout.

- Symmetric layout. In the symmetric layout of tourism toilets, most belong to this type. Natural symmetry forms the north island and south island or the east west, echo or up and down or so in order to achieve the core purpose of the convenient visitors go to the toilet. As a result, the symmetric layout of tourism toilets is more symmetrical in equilibrium.

\section{Conclusion}

In this paper, we analyze tourism toilet layout for Sichuan mountain resort based on graph theory. Tourism toilets have become important issues, it is because of its long cold, and the resulting large children's complaint, tourism, paid a high price for it. After several years of development, our country travel network all made a lot of improvement, but still there are less number, low grade, grade and management chaos, pollution and other issues. Tourism toilets should be in strict accordance with the standard construction as to further promote and improve the ecological environment of tourism toilets and speed up the construction funds of multiple beams way to emphasize the manifest "toilet" and pay attention to humanization. In the future research, we will integrate the concepts of the graph theory and more related techniques for further modification.

\section{Acknowledgements}

This paper is supported by the: Project on the humanities and Social Sciences (Tourism Research) of Sichuan Provincial Department of education in 2013(LYC13-17).

\section{References}

[1]Langer, Sebastian, and Adrijana Car. "GIS-based Decision Support for Public Toilet Site Selection: A Case Study of South Batinah Region in Oman." (2014): 135-139.

[2]Lang, Ling Yun, and Ke Xie. "The Analysis of Tourism Village's Residential Pattern." Applied Mechanics and Materials. Vol. 438. 2013.

[3]Lin, Wei-Cheng. "An ethnic enclave transformed into a tourism enclave under globalisation in Taiwan." International Journal of Liability and Scientific Enquiry 6.4 (2013): 247-256.

[4] Inouye, Carlos Eduardo Nakao, et al. "Modelling the spatial dynamics of urban growth and land use changes in the north coast of São Paulo, Brazil." Ocean \& Coastal Management 108 (2015).

[5] Bissell, William Cunningham. "When the film festival comes to (down) town: Transnational circuits, tourism, and the urban economy of images." Global Downtowns (2012): 160-185. 